\title{
Is the 2013 American Thoracic Society CPAP-tracking system algorithm useful for managing non-adherence in long-term CPAP-treated patients?
}

Marie-Caroline Rotty ${ }^{1,2}$, Jean-Pierre Mallet ${ }^{3}$, Carey M. Suehs ${ }^{3,4}$, Christian Martinez ${ }^{2}$, Jean-Christian Borel ${ }^{5}$, Claudio Rabec ${ }^{6}$, Arnaud Bourdin ${ }^{3,7}$, Nicolas Molinari ${ }^{1,4}$ and Dany Jaffuel ${ }^{3,8^{*}}$ (i)

\begin{abstract}
Background: Whereas telemedicine usage is growing, the only clinical algorithm for Continuous Positive Airway Pressure (CPAP) adherence management is that stipulated by the 2013 American Thoracic Society (ATS). The capacity of the latter to predict non-adherence in long-term CPAP-treated patients has not been validated.

Methods: Patients from the prospective real-life InterfaceVent study (NCT03013283, study conducted in an adult cohort undergoing at least 3 months of (PAP) and eligible for ATS algorithm usage were analysed. The residual device Apnea-Hypopnea-Index (AHI flow) and High Large Leak (HLL) thresholds proposed in the ATS algorithm were evaluated for predicting adherence (i.e. AH flow $>10 / \mathrm{h}$, HLLs 95th $>24 \mathrm{~L} /$ min for ResMed $^{\varpi}$ devices and ResMed ${ }^{\oplus}$ nasal mask, HLLs 95th > 36 I/min for ResMed ${ }^{\circledast}$ devices and ResMed ${ }^{\circledast}$ oronasal masks, HLLs $>1$ h for Philips ${ }^{\circledast}$ devices and HHLs $>60 \mathrm{l} / \mathrm{min}$ for Fisher \& Paykel $^{\oplus}$ devices). Adherence was defined according to the 2013 ATS algorithm (i.e. CPAP use $>4 \mathrm{~h} / \mathrm{j}$ for at least $70 \%$ of days).

Results: 650/1484 patients eligible for ATS algorithm usage were analysed (15.38\% non-adherent, $74 \%$ male with a median $\left(\mathrm{IQ}_{25-75}\right)$ age of $68(61-77)$ years, a body mass index of $30.8(27.7-34.5) \mathrm{kg} / \mathrm{m}^{2}$, an initial AHI of 39 (31-55) events/h, and CPAP-treatment-duration of 5.1 (2.2-7.8) years). Logistic regression analysis demonstrated no significant relationship between the ATS proposed AHlfow or HLL thresholds and non-adherence. Complementary ROC curve analysis failed to determine satisfactory AHl flow and HLL thresholds.
\end{abstract}

Conclusion: When managing non-adherence in long-term CPAP-treated patients, our data do not validate absolute AHI flow or HLL thresholds in general.

Trial registration: The INTERFACE-VENT study is registered on ClinicalTrials.gov (Identifier: study (NCT03013283).

Keywords: CPAP, Leaks, Apnea-hypopnea index, Telemedicine

\section{Background}

Continuous Positive Airway Pressure (CPAP) is the cornerstone of obstructive sleep apnea treatment. Previous studies have reported a correlation between patient adherence and treatment outcomes [1]. CPAP devices

\footnotetext{
* Correspondence: dany.jaffuel@wanadoo.fr

${ }^{3}$ Department of Respiratory Diseases, Montpellier University Hospital, Arnaud de Villeneuve Hospital, 371, Avenue Doyen Giraud, 34295 Montpellier Cedex 5, France

${ }^{8}$ Pulmonary Disorders and Respiratory Sleep Disorders Unit, Polyclinic

Saint-Privat, Boujan sur Libron, France

Full list of author information is available at the end of the article
}

can track adherence, but also leaks and residual ApneaHypopnea-Index $\left(\mathrm{AHI}_{\text {flow }}\right)$ values. CPAP tracking systems intuitively seem useful, but there are few data demonstrating the benefit of such systems in improving CPAP adherence [2]. In particular, the clinical significance of device-reported leaks or device reported $\mathrm{AHI}_{\text {flow }}$ is unknown. As underlined in the 2013 American Thoracic Society (ATS) statement, it was speculated that High Large Leaks (HLLs) and high AHI flow may affect CPAP adherence. Thus, HLLs and $\mathrm{AHI}_{\text {flow }}$ reported by manufacturers were cautiously included in the 2013 ATS clinical

(c) The Author(s). 2019 Open Access This article is distributed under the terms of the Creative Commons Attribution 4.0 International License (http://creativecommons.org/licenses/by/4.0/), which permits unrestricted use, distribution, and 
algorithm for using CPAP adherence tracking systems [3]. This statement is the only one available to clinicians, and despite increasing telemedicine usage in the field, it remains untested. In this context, the aim of this study is to assess the impact of HLLs and high $\mathrm{AHI}_{\text {flow }}$ on the adherence of long-term CPAP-treated patients in real-life conditions.

\section{Methods}

\section{Study design}

The InterfaceVent study is a prospective real-life study conducted in an adult cohort undergoing at least 3 months of CPAP for sleep apnea syndrome (SAS), defined according to the French Social Security system criteria: 1) Apnea Hypopnea Index (AHI) $\geq 30 / h$ (or $\mathrm{AHI} \geq 15 / \mathrm{h}$ and more than $10 / \mathrm{h}$ respiratory-effortrelated arousal), and 2) associated with sleepiness and $>3$ symptoms from among snoring, headaches, hypertension, reduced vigilance, libido disorders, nycturia). Following an initial prescription by one of the 336 device-prescribing physicians in the Occitanie region of France, these patients were provided care by the association "Apard", ADENE group, a non-profit home-care provider. Patient inclusions were performed during a home-visit by one of the 32
Apard technicians. Patients included in the analysis are those for whom it is possible to apply the 2013 ATS clinical algorithm for using CPAP adherence tracking systems (Fig. 1).

\section{ATS algorithm thresholds}

The $\mathrm{AHI}_{\text {flow }}$ threshold and HLLs thresholds chosen in the present paper are those proposed in the 2013 ATS algorithm (i.e. $\mathrm{AHI}_{\text {flow }}>10 / \mathrm{h}, \mathrm{HLL}$ 95th $>24 \mathrm{~L} / \mathrm{min}$ for ResMed devices and ResMed ${ }^{\circ}$ nasal mask, HLL 95th $>36 \mathrm{l} / \mathrm{min}$ for ResMed $^{\oplus}$ devices and ResMed ${ }^{\circ}$ oronasal masks, HLL $>1 \mathrm{~h}$ of large leaks for Philips ${ }^{\circ}$ devices and HHL $>60 \mathrm{l} / \mathrm{min}$ for Fisher \& Paykel $^{\circ}$ devices whatever the type and brand of the interface). Adherence was also defined according to the 2013 algorithm (CPAP use $>4 \mathrm{~h} / \mathrm{j}$ at least $70 \%$ of days).

\section{Collected data}

In addition to demographic/clinical data and the mask/ device data, the response to the Epworth Sleepiness Scale (ESS) and the Euroqol 5 Dimensions 3 level version (EQ-5D-3 L) questionnaires were also collected. Patient perceptions of leaks and mouth dryness were assessed using an 11-point visual analogue scale (VAS)

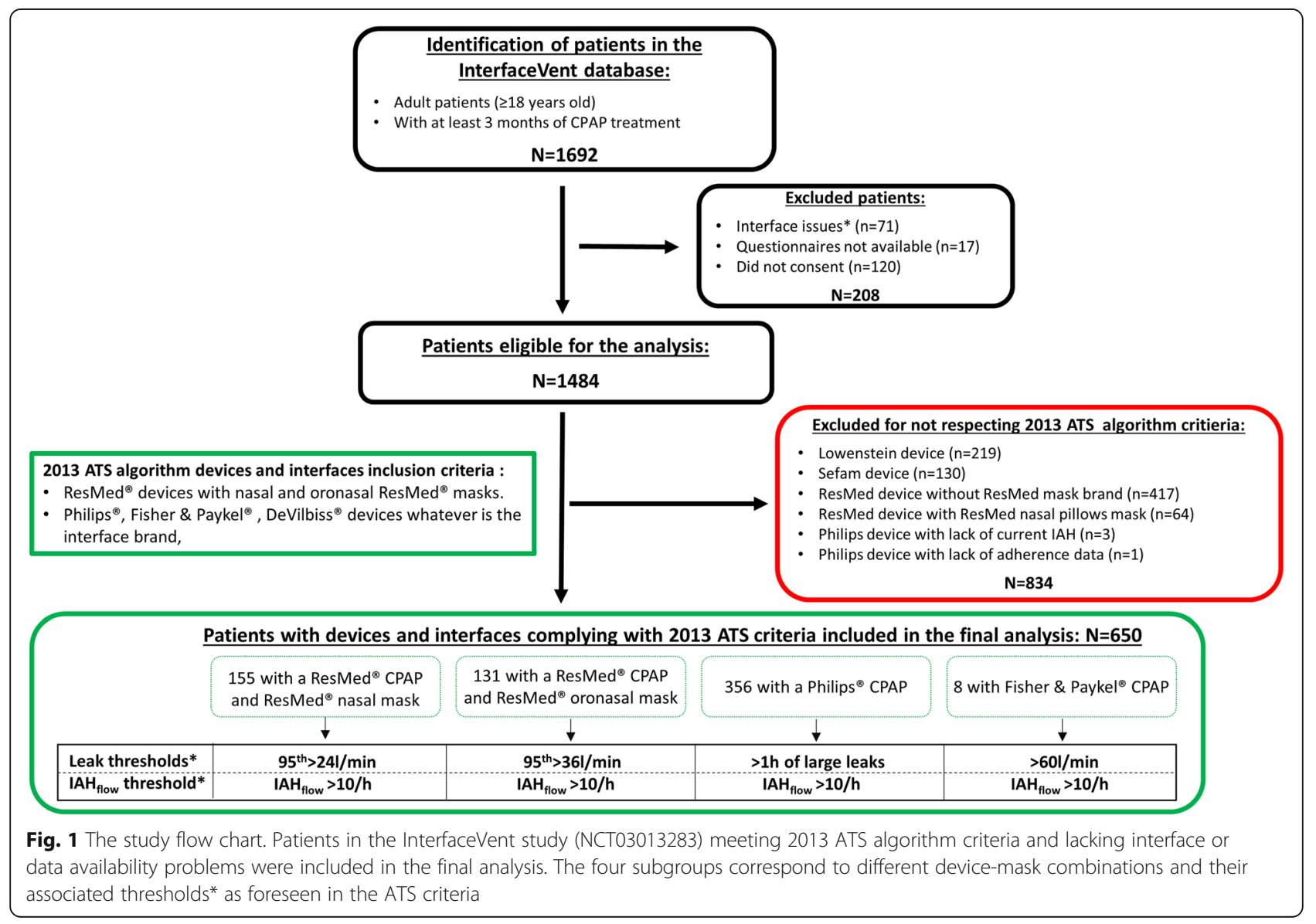


(ranging from no discomfort (0) to very uncomfortable (10)).

\section{Statistical analyses}

Multivariable logistic regression analyses were used to study associations between adherence and collected data. Using forward-stepwise selection, covariates with a univariable $p$-value $<0.15$ were fed into multivariable analyses. Odds-ratios with their $95 \%$ CI were calculated according to Woolf's method, with an alpha risk of 0.05 . Model goodness-of-fit was assessed using the HosmerLemeshow test. Receiver operating curves (ROC) were created to determine $\mathrm{AHI}_{\text {flow }}$ and HLL thresholds predictive of non-adherence, as determined by maximizing the Youden index.

\section{Results}

Six hundred fifty patients were analysed: 155 with a ResMed $^{\circ}$ CPAP and ResMed $^{\circ}$ nasal mask, 131 with a ResMed $^{\circ}$ device and ResMed $^{\circ}$ oronasal mask, 356 with a Philips ${ }^{\circ}$ device regardless of the interface used and 8 with Fisher \& Paykel Device. The results of the logistic regression analysis with adherence as the dependent variable are summarized in Table 1.

Univariable analysis failed to demonstrate a significant HLL or $\mathrm{AHI}_{\text {flow }}$ effect on adherence (Table 1). Interestingly, the VAS for leaks was not associated with non-adherence in the univariable analysis, whereas mouth dryness was $(p=0.019)$. Finally, multivariable logistic regression demonstrated that increased body mass index or the presence of a partner was significantly positively associated with adherence.

In order to re-evaluate $\mathrm{AHI}_{\text {flow }}$ and HLL thresholds associated with non-adherence, we generated ROCs curves. For ResMed ${ }^{\circ}$-reported $\mathrm{AHI}_{\text {flow }}$, accuracy was low (area under curve (AUC) of 0.53 [0.44-0.62]) for a threshold of $13.3 / \mathrm{h}$ (sensitivity was 0.04 and specificity was 0.99 , positive predictive value (PPV) was 0.50 and negative predictive value (NPV) was 0.85 ). For Philips reported $\mathrm{AHI}_{\text {flow }}$, accuracy was low (AUC of 0.51 [0.43$0.60]$ ) for a threshold of $1.4 / \mathrm{h}$ (sensitivity was 0.87 and specificity was 0.21 , PPV was 0.17 and NPV) was 0.90 . For 95th ResMed ${ }^{\circ}$ nasal leaks, accuracy was low with an AUC of 0.62 [0.49-0.75] for a threshold of $18 \mathrm{~L} / \mathrm{min}$ (sensitivity was 0.77 and specificity was 0.45 , PPV was 0.19 and NPV was 0.92). For 95th ResMed $^{\circ}$ oronasal leaks, accuracy was low (AUC of 0.58 [0.45-0.72]) for a threshold of $15.6 \mathrm{~L} / \mathrm{min}$ (sensitivity was 0.65 and specificity was 0.51 , PPV was 0.22 and NPV was 0.87 ). The HLL Philips ${ }^{\circ}$ ROC curve could not be created because of a Hosmer and Lemeshow positive test, indicating invalid (ROC) values. We did not generate $\mathrm{AHI}_{\text {flow }}$ and HLL ROC curves for the 8 adherent patients treated with Fisher \& Paykel $^{\circ}$ devices.

\section{Discussion}

The 2013 American Thoracic Society statement [3] on the CPAP device tracking systems underlined the absence of standards for scoring flow signals, or measuring mask leaks, as well as the non-existence of standards on how to use these data in order to improve outcomes. Our analysis suggests that in long-term CPAP-treated patients, the 2013 ATS statement proposed thresholds for HLLs and $\mathrm{AHI}_{\text {flow }}$ are not associated with non-adherence. In addition, we failed to find statistically satisfactory $\mathrm{AHI}_{\text {flow }}$ and HLL thresholds for predicting non-adherence.

\section{AHI flow thresholds}

As underlined by the 2013 ATS statement, $\mathrm{AHI}_{\text {flow }}$ is not a true surrogate of AHI measured by polysomnography $\left(\mathrm{AHI}_{\mathrm{PSG}}\right)$. Indeed, previous studies have reported that $\mathrm{AHI}_{\text {flow }}$ was not always correlated or concordant with $\mathrm{AHI}_{\mathrm{PSG}}$ and major differences exist between manufacturer definitions of the residual events $[4,5]$. In this regard, different ROC-determined $\mathrm{AHI}_{\text {flow }}$ thresholds were found for different machines (as can be expected, considering that device manufacturers all use different event definitions [3]). Similarly, in short-term treated patients, Valentin et al. reported that Philips'-reported $\mathrm{AHI}_{\text {flow }}$ during the first week of treatment was associated with lower adherence to CPAP therapy at 5 weeks of treatment, but the authors were unable to propose an $\mathrm{AHI}_{\text {flow }}$ threshold [6].

\section{Leak thresholds}

Our long-term study agrees with two other short-term studies focused on leaks. Valentin et al. demonstrated that device-reported leaks during the first week of treatment were slightly associated with lower adherence to CPAP therapy at 5 weeks of treatment (a threshold-adjusted leak-level of $4.9 \mathrm{~L} / \mathrm{min} / \mathrm{cm} \mathrm{H}_{2} \mathrm{O}$ was associated with a sensitivity of 0.62 and specificity of 0.65 for discriminating adherent and non-adherent patients) [6]. Baltzan et al. reported (using a manual score of devicereported leaks with a cut-off of $20 \mathrm{l} / \mathrm{min}$ of unintentional leaks and patterns of continuous leaks or serrated leaks) that the highest quartile of percentage time in continuous leaks may be linked to adherence during the first 3 months of treatment (but the relationship did not reach statistical significance) [7]. The aetiology of leaks is also an important issue, and more attention should be given to mouth leaks, as recommended by the 2013 ATS statement [3]. Bachour et al. have reported that mouth breathing patients were less adherent to CPAP-treatment at 3 months [8]. In this regard, the fact that the present study indicates that the mouth dryness VAS score is associated with lower adherence during univariable analysis is quite interesting. Mouth dryness may potentially 
Table 1 Logistic regression analysis with adherence (>4h /day, 70\% of the days) as the dependent variable

\begin{tabular}{|c|c|c|c|c|c|c|c|}
\hline & Whole population & $\begin{array}{l}\text { Non adherent } \\
N=100\end{array}$ & $\begin{array}{l}\text { Adherent } \\
N=550\end{array}$ & Crude OR [95\% Cl] & $p$-value & $\begin{array}{l}\text { Adjusted OR } \\
{[95 \% \mathrm{Cl}]}\end{array}$ & $p$-value \\
\hline \multicolumn{8}{|l|}{ Demographics } \\
\hline Age (yrs) & $68[61 ; 74]$ & $68[60 ; 75]$ & $68[61 ; 74]$ & $1.00[0.98 ; 1.02]$ & 0.80 & & \\
\hline Gender, female (\%) & 173/650 (26.6) & $32 / 100(32.0)$ & $141 / 550(25.6)$ & $0.73[0.46 ; 1.16]$ & 0.19 & & \\
\hline BMI $\left(\mathrm{kg} / \mathrm{m}^{2}\right)$ & $30.8[27.7 ; 34.5]$ & $29.4[26.5 ; 32.3]$ & $31.1[27.8 ; 34.9]$ & $1.07[1.02 ; 1.12]$ & 0.003 & $1.07[1.01 ; 1.13]$ & 0.03 \\
\hline Initial AHI (event/h) & $39[31 ; 55]$ & $37.9[30.0 ; 52.0]$ & $39.0[31.0 ; 57.0]$ & $1.01[0.99 ; 1.02]$ & 0.16 & & \\
\hline Active smokers (\%) & 77/637 (12.1] & 13/99 (13.1) & $64 / 538(11.9)$ & $0.89[0.47 ; 1.69]$ & 0.73 & & \\
\hline Beard (\%) & 75/456 (16.5) & 10/64 (15.6) & 65/392 (16.6) & $1.05[0.50 ; 2.18]$ & 0.70 & & \\
\hline Mustache (\%) & $42 / 456(9.2)$ & $7 / 64(10.9)$ & 35/392 (8.9) & $0.81[0.34 ; 1.92]$ & 0.60 & & \\
\hline No mustache no beard (\%) & $339 / 456(74.3)$ & 47/64 (73.4) & 292/392 (74.5) & Ref & 0.88 & & \\
\hline Presence of partner & $457 / 640(71.4)$ & $61 / 96(63.5)$ & $396 / 544(72.8)$ & $1.54[0.97 ; 2.42]$ & 0.07 & $2.03[1.18 ; 3.50]$ & 0.011 \\
\hline Active workers & 130/632 (20.6) & 20/95 (21.1) & $110 / 537(20.5)$ & $0.97[0.57 ; 1.65]$ & 0.90 & & \\
\hline ESS (VAS score) & $5[3 ; 8]$ & $6[3 ; 8]$ & $5[3 ; 9]$ & $0.99[0.95 ; 1.05]$ & 0.87 & & \\
\hline \multicolumn{8}{|l|}{ EQ-5D-3 L } \\
\hline Problems with mobility (\%) & $157 / 623(25.2)$ & 25/94 (26.6) & $132 / 529(26.6)$ & $0.92[0.58 ; 1.51]$ & 0.74 & & \\
\hline Problems with self-care (\%) & $38 / 617(6.2)$ & 6/94 (6.38) & $32 / 523(6.12)$ & $0.96[0.39 ; 2.35]$ & 0.92 & & \\
\hline Problems with usual activities (\%) & $124 / 620(20.0)$ & 20/96 (20.8) & 104/524 (19.9) & $0.94[0.55 ; 1.61]$ & 0.82 & & \\
\hline Problems of pain/discomfort (\%) & $347 / 623(55.7)$ & $54 / 96(56.3)$ & 293/527 (55.6) & $0.97[0.63 ; 1.51]$ & 0.91 & & \\
\hline Problems of anxiety/depression (\%) & $240 / 624(38.5)$ & $41 / 96(42.7)$ & 199/528 (37.9) & $0.81[0.52 ; 1.26]$ & 0.35 & & \\
\hline EQ-5D-3 L Health (VAS score) & $69.8[50.2 ; 80.1]$ & $69.2[49.4 ; 80.0]$ & $69.9[50.4 ; 80.2]$ & $1.01[0.99 ; 1.02]$ & 0.17 & & \\
\hline \multicolumn{8}{|l|}{ Device } \\
\hline Treatment duration (yrs) & $4.9[2.1 ; 9.8]$ & $3.8[1.3 ; 8.1]$ & $5.1[2.3 ; 10.2]$ & $1.06[1.01 ; 1.11]$ & 0.02 & & \\
\hline Fixed pressure (\%) & $91 / 650(14.0)$ & $12 / 100(12.0)$ & $79 / 550(14.4)$ & $1.23[0.64 ; 2.35]$ & 0.53 & & \\
\hline 90th/95th Pressure $\left(\mathrm{cmH}_{2} \mathrm{O}\right)$ & $10.0[8.30 ; 11.8]$ & $9.9[8.0 ; 11.5]$ & $10.0[8.3 ; 11.8]$ & $1.10[0.99 ; 1.21]$ & 0.09 & & \\
\hline Oronasal (\%) & 216/650 (33.2) & 43/100 (43.0) & $173 / 550(31.5)$ & $0.58[0.37 ; 0.91]$ & 0.14 & & \\
\hline Nasal (\%) & $375 / 650(57.7)$ & $47 / 100(47.0)$ & $328 / 550(59.6)$ & Ref & 0.07 & & \\
\hline Nasal pillows (\%) & $59 / 650(9.1)$ & 10/100 (10.0) & $49 / 550(8.91)$ & $0.70[0.33 ; 1.48]$ & 0.83 & & \\
\hline Heated humidifier & $386 / 650(59.4)$ & $66 / 100(66.0)$ & $320 / 550(58.2)$ & $0.72[0.46 ; 1.12]$ & 0.14 & & \\
\hline Heated breathing tube & $22 / 650(3.4)$ & $3 / 100(3.0)$ & $19 / 550(3.5)$ & $1.16[0.34 ; 3.99]$ & 0.82 & & \\
\hline \multicolumn{8}{|l|}{2013 ATS statement tested thresholds } \\
\hline Current $\mathrm{AH}_{\text {flow }}(>10)$ & $23 / 650(3.4)$ & $5 / 100(5.0)$ & $17 / 550(3.1)$ & $0.61[0.22 ; 1.68]$ & 0.34 & & \\
\hline ResMed Nasal large leaks (95th > 24 L) & $53 / 154(34.4)$ & $10 / 22(45.5)$ & 43/132 (32.6) & $0.58[0.23 ; 1.45]$ & 0.24 & & \\
\hline ResMed Facial large leaks (95th > 36 L) & 28/131 (21.4) & 8/23 (34.8) & 20/108 (18.5) & $0.43[0.16 ; 1.14]$ & 0.09 & & \\
\hline Philips leaks (> $1 \mathrm{~h}$ of large leaks) & $11 / 356(3.1)$ & $1 / 55(1.82)$ & 10/301 (3.32) & $1.86[0.23 ; 14.8]$ & 0.56 & & \\
\hline Fisher Paykel leaks (> $60 \mathrm{~L} / \mathrm{min}$ ) & $8 / 8(100)$ & 0/8 (0) & $8 / 8(100)$ & NA & & & \\
\hline \multicolumn{8}{|l|}{ VAS scores } \\
\hline Patient perceived leaks (VAS score) & $3[1 ; 5]$ & $3.0[1.0 ; 6.0]$ & $3.0[0.0 ; 5.0]$ & $0.97[0.91 ; 1.05]$ & 0.48 & & \\
\hline $\begin{array}{l}\text { Patient perceived mouth dryness } \\
\text { (VAS score) }\end{array}$ & $3[0 ; 7]$ & $5[1 ; 8]$ & $3[0 ; 7]$ & $0.93[0.88 ; 0.99]$ & 0.019 & & \\
\hline
\end{tabular}

be the consequence of mouth leaks, although we cannot overcome other confounding factors in our study (medical prescriptions and co-morbidities) [9] that help explain the absence of significance at the multivariable level. A VAS score is not sufficient for the accurate evaluation of mouth opening and new tools are required. Recently, the suitability of a mandibular movement sensor for evaluating mouth opening effects on unintentional leaks was demonstrated [10] and may respond to this need [11]. 


\section{Study limits}

Beyond these negative results, it is important to remember that our population was treated on a long-term basis and our conclusions may not be applicable to short-term situations. For long-term patients, in contrast with absolute-value thresholds, the percentage-change may be of greater interest [12]. However, our study design did not enable us to test this hypothesis.

\section{Conclusions}

Six years after the 2013 ATS statement and during a time when telemedicine is growing, our data suggest that before proceeding with remote monitoring initiatives, it is necessary to validate the diagnostic potential of data generated by CPAP tracking systems before they are implicated in a decision making process.

\section{Abbreviations \\ $\mathrm{AHI}_{\text {flow: }}$ Residual Apnea-Hypopnea-Index; ATS: American Thoracic Society; AUC: Area Under Curve; BMI: Body Mass Index; CPAP: Continuous Positive Airway Pressure; EQ-5D-3 L: Euroqol 5 Dimensions 3 level version; ESS: Epworth Sleepiness Scale; HLLs: High Large Leaks; VAS: Visual Analog Scale}

\section{Acknowledgements}

The InterfaceVent investigators gratefully recognise the vital input and support of the non-profit home care provider APARD and the ADENE group. Administrative Team: Bernard Alsina, Valérie Bachelier, Pierre Coulot, Christophe Jeanjean, Philippe Lansard, Joël Nogue.

Technician Team: Matthieu Alberti, Julien Bauchu, Yannick Baudelot, Gregory Bel, Julien Bernard, Julien Bourrel, Frédéric Bousquet, David Crespy, Olivier Cubero, Eric Deghal, Fabien Deville, Sébastien Faure, Laure Ferraz, Olivier Gaubert, Georges Guichard, Franck Issert, Renaud Lopez, Mounia Maachou, Clément Maurin, David Minguez, Fabien Moubeche, Christophe Pinotti, Liva Ranaivo, Lazhar Saighi, Frédéric Sola, Frédéric Tallavignes, Olivier Tramier, Jean-Michel Tribe, Jean-Marc Uriol, Romain Vernet.

\section{Authors' contributions}

DJ access to the data and takes responsibility for the integrity and accuracy of the analysis. All authors contributed to and approved the final submitted manuscript. MCR: study design, data collection, analysis, and manuscript preparation; JPM: study design and manuscript preparation, CMS: study design and manuscript preparation, CM: study design, data collection, JCB: analysis, manuscript preparation, CR: analysis, manuscript preparation, $A B$ : study design, analysis and manuscript preparation, NM: study design, data collection, analysis, and manuscript preparation, DJ: study design, data collection, analysis, and manuscript preparation.

\section{Funding}

The authors have no support nor funding to report in relation with the present study.

\section{Availability of data and materials}

The datasets used and/or analyzed during the current study are available from the corresponding author on reasonable request.

\section{Ethics approval and consent to participate}

This study presents an analysis of the InterfaceVent study registered on ClinicalTrials.gov (NCT03013283). The protocol was reviewed and approved by an independent ethics committee (Comité de Protection des Personnes Sud Mediterranée 1; reference number RO-2016/50).

\section{Consent for publication}

Not applicable.

\section{Competing interests}

The authors declare that they have no competing interests.

\section{Author details}

"IMAG, CNRS, Montpellier University, Montpellier University Hospital, Montpellier, France. ${ }^{2}$ Apard groupe Adène, Montpellier, France. ${ }^{3}$ Department of Respiratory Diseases, Montpellier University Hospital, Arnaud de Villeneuve Hospital, 371, Avenue Doyen Giraud, 34295 Montpellier Cedex 5, France. ${ }^{4}$ Department of Medical Information, Montpellier University Hospital, Montpellier, France. ${ }^{5}$ Grenoble Alps University, Inserm U1042, HP2 (Hypoxia PhysioPathology) Laboratory, Centre Hospitalier Universitaire Grenoble Alpes, Grenoble, France. ${ }^{6}$ Pulmonary Department and Respiratory Critical Care Unit, University Hospital Dijon, Dijon, France. ${ }^{7}$ PhyMedExp (INSERM U 1046, CNRS UMR9214), Montpellier University, Montpellier, France. ${ }^{8}$ Pulmonary Disorders and Respiratory Sleep Disorders Unit, Polyclinic Saint-Privat, Boujan sur Libron, France.

Received: 8 May 2019 Accepted: 31 July 2019

Published online: 12 September 2019

\section{References}

1. Yu J, Zhou Z, McEvoy RD, Anderson CS, Rodgers A, Perkovic V, et al. Association of Positive Airway Pressure with Cardiovascular Events and Death in adults with sleep apnea: a systematic review and meta-analysis. JAMA. 2017:318:156-66.

2. Hwang D, Chang JW, Benjafield AV, Crocker ME, Kelly C, Becker KA, et al. Effect of telemedicine education and Telemonitoring on continuous positive airway pressure adherence. The tele-OSA randomized trial. Am J Respir Crit Care Med. 2018;197:117-26.

3. Schwab RJ, Badr SM, Epstein LJ, Gay PC, Gozal D, Kohler M, et al. An official American Thoracic Society statement: continuous positive airway pressure adherence tracking systems. The optimal monitoring strategies and outcome measures in adults. Am J Respir Crit Care Med. 2013;188:613-20.

4. Huang H-CC, Hillman DR, McArdle N. Control of OSA during automatic positive airway pressure titration in a clinical case series: predictors and accuracy of device download data. Sleep. 2012;35:1277-1283A.

5. Reiter J, Zleik B, Bazalakova M, Mehta P, Thomas RJ. Residual events during use of CPAP: prevalence, predictors, and detection accuracy. J Clin Sleep Med. 2016;12:1153-8.

6. Valentin A, Subramanian S, Quan SF, Berry RB, Parthasarathy S. Air leak is associated with poor adherence to autoPAP therapy. Sleep. 2011;34:801-6.

7. Baltzan MA, Dabrusin R, Garcia-Asensi A, Sully J-L, Parenteau M, Tansimat G, et al. Leak profile inspection during nasal continuous positive airway pressure. Respir Care. 2011;56:591-5.

8. Bachour A, Maasilta P. Mouth breathing compromises adherence to nasal continuous positive airway pressure therapy. Chest. 2004;126:1248-54.

9. Rakotonanahary D, Pelletier-Fleury N, Gagnadoux F, Fleury B. Predictive factors for the need for additional humidification during nasal continuous positive airway pressure therapy. Chest. 2001;119:460-5.

10. Lebret M, Arnol N, Martinot J-B, Lambert L, Tamisier R, Pepin J-L, et al. Determinants of unintentional leaks during CPAP treatment in OSA. Chest. 2018;153:834-42.

11. Genta PR, Lorenzi-Filho G. Sealing the leak: a step forward in improving CPAP adherence. Chest. 2018;153:774-5.

12. Borel J-C, Pelletier J, Taleux N, Briault A, Arnol N, Pison C, et al. Parameters recorded by software of non-invasive ventilators predict COPD exacerbation: a proof-of-concept study. Thorax. 2015;70:284-5.

\section{Publisher's Note}

Springer Nature remains neutral with regard to jurisdictional claims in published maps and institutional affiliations. 